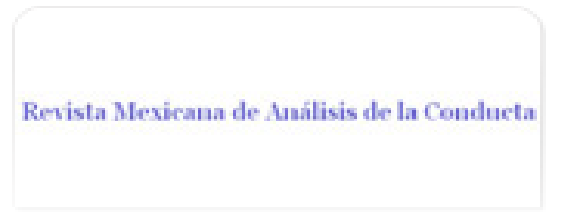

Revista Mexicana de Análisis de la Conducta ISSN: 0185-4534

editora@rmac-mx.org

Sociedad Mexicana de Análisis de la Conducta México

Ruiz, Jorge A.; Bruner, Carlos A.

Transformación de un programa de intervalo fijo de reforzamiento con agua en un procedimiento de beber inducido por el programa

Revista Mexicana de Análisis de la Conducta, vol. 31, núm. 1, junio, 2005, pp. 47-65

Sociedad Mexicana de Análisis de la Conducta

Guadalajara, México

Disponible en: http://www.redalyc.org/articulo.oa?id=59331103

Cómo citar el artículo

- Número completo

- Más información del artículo

Página de la revista en redalyc.org

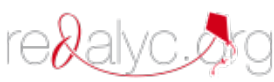

Sistema de Información Científica

Red de Revistas Científicas de América Latina, el Caribe, España y Portugal Proyecto académico sin fines de lucro, desarrollado bajo la iniciativa de acceso abierto 


\title{
TRANSFORMACIÓN DE UN PROGRAMA DE INTERVALO FIJO DE REFORZAMIENTO CON AGUA EN UN PROCEDIMIENTO DE BEBER INDUCIDO POR EL PROGRAMA
}

\author{
TRANSFORMATION OF A FIXED-INTERVAL SCHEDULE \\ OF WATER REINFORCEMENT INTO ONE PROCEDURE OF \\ SCHEDULE-INDUCED DRINKING
}

JORGE A. RUIZ Y CARLOS A. BRUNER ${ }^{1}$

UNIVERSIDAD NACIONAL AUTÓNOMA DE MÉXICO

\begin{abstract}
RESUMEN
Para determinar si los efectos específicos de las variables que controlan el beber inducido por el programa son ajenos o no al campo del condicionamiento, en el presente estudio se añadieron una a una la privación de comida, la entrega periódica de comida y la entrega continua de agua a un programa de reforzamiento con agua de intervalo fijo, hasta transformar este procedimiento operante en uno de beber inducido por el programa. En la primera condición experimental tres ratas privadas de agua lengüetearon un tubo vacío para obtener una gota de agua conforme a un programa de reforzamiento de intervalo fijo $64 \mathrm{~s}$. Una vez que el lengüeteo se estabilizó en un patrón de festón durante el intervalo entre reforzadores, se añadieron las variables del beber inducido por el programa. El patrón de festón cambió progresivamente hasta convertirse en una función en forma de $U$ invertida, típica del beber inducido por el programa. La transición progresiva entre ambos patrones de
\end{abstract}

1 Nota de los autores: El presente estudio se realizó con el apoyo del subsidio 35011-H otorgado por el Consejo Nacional de Ciencia y Tecnología (CONACYT) al segundo autor. Los datos reportados sirvieron como Tesis de Licenciatura para el primer autor. Los autores agradecen el constante apoyo del Dr. Raúl Avila en la elaboración del presente trabajo. Dirigir correspondencia al segundo autor: Laboratorio de Condicionamiento Operante, Facultad de Psicología, UNAM. Av. Universidad 3004, Cd. Universitaria. México, D.F. 04510. Email: bruner@servidor.unam.mx. 
respuesta mostró que las variables independientes nunca dejaron de reflejar efectos ampliamente conocidos en la literatura sobre condicionamiento y motivación. Por lo tanto, parece injustificado categorizar al beber inducido por el programa como un fenómeno anómalo.

Palabras clave: Beber inducido por el programa, condicionamiento, motivación, ratas.

\begin{abstract}
To determine whether or not the specific effects of the schedule-induced drinking variables are anomalous to conditioning, in the present study food deprivation, periodic food delivery and continuous water delivery were added one by one to a fixed-interval schedule of water reinforcement, until this operant procedure was transformed into one of schedule-induced drinking. In the first experimental condition three water-deprived rats licked an empty tube to obtain a drop of water according to 64-s fixed-interval schedule of reinforcement. Once licking stabilized into a scalloped pattern during the interreinforcement interval, the schedule-induced drinking variables were added. The scalloped licking pattern was transformed progressively into an inverted U-function, typical of schedule-induced drinking. The progressive transition between both response patterns showed that the independent variables never ceased to reflect widely known effects in both, the motivation and conditioning literatures. Therefore, it seems unjustified to categorize schedule-induced drinking as a phenomenon anomalous to conditioning.

Keywords: Schedule-induced drinking, conditioning, motivation, rats.
\end{abstract}

Durante los últimos 40 años el beber inducido por el programa (BIP, en adelante) ha aparentado ser un fenómeno inexplicable por los principios del análisis experimental de la conducta. Desde que Falk (1961) reportó que ratas privadas de comida expuestas a un programa de reforzamiento aperiódico con comida consumían cantidades sustanciales de agua durante las sesiones experimentales se han llevado a cabo diferentes intentos por tratar de controlar la ocurrencia del BIP con procedimientos de condicionamiento $\mathrm{Pa}-$ vloviano y de condicionamiento operante.

Respecto a los estudios en los que se trató de condicionar la ocurrencia del BIP mediante la presentación de un estímulo asociado a la comida, algunos autores reportaron la ocurrencia del BIP ante presentaciones aisladas de un estímulo asociado a la comida (e.g., Corfield-Sumner, Blackman, \& Stainer, 1977) mientras que otros autores reportaron hallazgos contradictorios (e.g., Allen \& Porter, 1977; Allen, Porter, \& Araize, 1975). En cuanto al condicionamiento operante del BIP, algunos autores sugirieron que si el BIP 
era operante su ocurrencia estaría modulada por sus consecuencias. Dado que el BIP no tiene consecuencias programadas experimentalmente su ocurrencia estaría controlada por la comida, reforzando de manera adventicia los lengüetazos al tubo con agua (cf., Wetherington, 1982). Sin embargo, la hipótesis de que el BIP podría tratarse de conducta supersticiosa reforzada accidentalmente por la comida (Clark, 1962) no prosperó dado que el BIP ocurría aun cuando se programaban demoras entre la comida y el último lengüetazo al tubo con agua (Falk, 1964; Segal \& Oden, 1969).

La falta de consistencia entre los estudios que trataron de condicionar al BIP con procedimientos Pavlovianos y entre los estudios que caracterizaron al BIP como una conducta supersticiosa, culminó en la conclusión de que el BIP debía tratarse de una tercera clase de conducta, diferente de la operante y de la respondiente (Falk, 1969, 1971, 1998; Wetherington, 1982). Incluso, algunos teóricos del aprendizaje contemporáneos han considerado que el fenómeno del BIP representa otro ejemplo más de la insuficiencia de los principios conocidos en el condicionamiento así como de la necesidad de crear nuevos conceptos ya sean de tipo cognoscitivo o bien mecanismos regulatorios para explicar la conducta (e.g., Breland \& Breland, 1961; Mowrer \& Klein, 1989; Timberlake, 1993, 1997, 2001).

Aun con el permanente desacuerdo entre los investigadores acerca de la naturaleza del BIP, se determinó que las principales variables que controlan la ocurrencia y magnitud del fenómeno eran la privación de comida y la entrega periódica de comida (Falk, 1969). Respecto a la privación de comida, en la literatura acerca del BIP la manipulación de diferentes niveles de privación de comida ha resultado en efectos claros y consistentes. El consumo de agua es mayor conforme aumenta el nivel de privación de comida (Falk, 1969; Keehn, 1979; Roper \& Nieto, 1979). Respecto al programa de entrega de comida, alargar la duración del intervalo entre comidas de 2 a $180 \mathrm{~s}$ resulta en un aumento en el consumo de agua y alargar la duración del intervalo hasta 300 s resulta en una disminución en el consumo de agua (Falk, 1966; Flory,1971; Hawkins, Schrot, Githens, \&Everett, 1972).

Es importante resaltar que las variables que controlan el BIP se han explorado exponiendo a los sujetos privados de comida a un programa de entrega de comida a intervalos de tiempo para establecer el BIP y posteriormente se ha manipulado una de estas variables mientras la otra se mantiene constante. Quizá el carácter anómalo que se le ha concedido al BIP resulta del efecto específico de combinar simultáneamente estas variables independientes, por lo que en el presente estudio se añadieron una a una las condiciones del BIP a un programa de reforzamiento con agua de intervalo fijo, hasta transformar el procedimiento operante en un procedimiento de beber inducido por el programa. El propósito del estudio fue reconocer el efecto de 
cada una de las variables del BIP sobre el consumo de agua y su distribución temporal como semejantes o ajenos al campo del condicionamiento.

\section{MÉTODO}

\section{Sujetos}

Se utilizaron tres ratas Wistar macho de tres meses de edad y experimentalmente ingenuas al inicio de la investigación. Se alojó a los sujetos en cajas habitación individuales con acceso controlado a la comida y al agua de acuerdo a cada una de las condiciones experimentales.

\section{Aparatos}

Se utilizó una cámara experimental (MED Assoc. Inc. Mod. ENV-007) que se colocó dentro de un cubículo sonoamortiguado (MED Assoc. Inc. Mod. ENV018) equipado con un ventilador para facilitar la circulación del aire dentro de la cámara experimental y un generador de ruido blanco (MED Assoc. Inc. Mod. ENV-225SM) para enmascarar cualquier ruido ajeno al experimento.

Al centro del panel frontal, $5 \mathrm{~cm}$ arriba del piso de la cámara, se colocó un recipiente para bolitas de comida (MED Assoc. Inc. Mod. ENV-200R1AM) y a la izquierda del recipiente se colocó una base de plástico (MED Assoc. Inc. Mod. ENV-250RM) sobre la cual se montó un tubo metálico conectado a una botella con agua. En la parte superior de la pared frontal, $1.5 \mathrm{~cm}$ abajo del techo de la cámara, se colocó un foco de $28 \mathrm{~V}$ (MED Assoc. Inc. Mod. ENV-215M) para iluminar la cámara experimental durante las sesiones. Una válvula de usos múltiples (Parker Hannitin Corporation Mod. VAC-20 PSIG) conectada a una botella con agua entregó $0.1 \mathrm{~mL}$ de agua en el tubo metálico cuando se operó con un pulso de $28 \mathrm{~V}$ durante $1 \mathrm{~s}$. Un dispensador de bolitas de comida (MED Assoc. Inc. Mod. ENV-203IR) entregó una bolita de comida de $25 \mathrm{mg}$ en el recipiente para la comida, en cada operación. Las bolitas de comida se fabricaron remoldeando comida pulverizada de la marca Harland Teklad. Se usó un contador de lengüetazos (MED Associates Inc. Mod. ENV250) para registrar los lengüetazos al tubo mediante el cierre de un circuito cada vez que el sujeto hacía contacto con el tubo. Se colocó una lámina de metal con un orificio de $13 \mathrm{~mm}$ de diámetro en el centro, $6 \mathrm{~mm}$ delante del tubo, para evitar que las ratas hicieran contacto con el tubo con cualquier otra parte del cuerpo que no fuera la lengua.

Se empleó una computadora con software MED-PC conectada a la cámara experimental mediante una interfase (MED Associates Inc. Mod. SG- 
503) para controlar los eventos experimentales y para registrar los lengüetazos de los sujetos.

\section{Procedimiento}

Con el fin de contar con un patrón conductual reconocible que sirviera de línea base para establecer los efectos de las variables del BIP en la primera condición se privó de agua a los sujetos durante 22 horas y sin ningún entrenamiento se les expuso a un programa de reforzamiento con agua de intervalo fijo (IF) $64 \mathrm{~s}$. Durante esta condición y en las posteriores se reforzaron los lengüetazos a un tubo vacío entregando $0.1 \mathrm{~mL}$ de agua en el mismo tubo cada vez que los sujetos cumplían el requisito del programa. Se usó un programa de reforzamiento con agua de IF $64 \mathrm{~s}$ debido a que se ha documentado el establecimiento de una respuesta sin entrenamiento bajo esta duración (e.g., Bruner \& Avila, 2002). Se decidió separar la conducta instrumental del lengüeteo a un tubo y la consecuencia de dicho lengüeteo, es decir, la obtención de una gota de agua debido a que Bruner y Avila sugirieron que en los procedimientos de BIP cada lengüetazo a un tubo se reforzaba con una gota de agua, conforme a un programa de reforzamiento continuo.

Dada la demostración de que la privación de comida es una variable necesaria para el establecimiento del BIP, en la segunda condición experimental se privó de comida a los sujetos para mantenerlos al $80 \%$ de su peso en alimentación libre. La privación de agua y el programa de reforzamiento con agua de IF 64 s se mantuvieron constantes.

La segunda variable que se determinó como necesaria para establecer el BIP es la entrega periódica de comida en la cámara experimental, por lo que en la tercera condición se añadió la entrega de comida $48 \mathrm{~s}$ después de cada entrega de agua (i.e., conforme a un programa de tiempo fijo (TF) $64 \mathrm{~s}$ fuera de fase $16 \mathrm{~s}$ respecto del programa de IF $64 \mathrm{~s}$ por agua). El programa de reforzamiento con agua de IF 64 s y la privación de agua y de comida se mantuvieron constantes.

En la mayoría de los estudios del BIP el agua se ha entregado conforme a un programa de reforzamiento continuo, así que durante la cuarta condición del estudio se acortó la duración del programa de reforzamiento con agua de IF de 64 a $2 \mathrm{~s}$, mientras la entrega de comida y la privación de agua y de comida se mantuvieron constantes. La razón para utilizar un IF corto en lugar del programa de reforzamiento continuo comúnmente usado en los estudios sobre el BIP, fue permitir que los sujetos consumieran totalmente la gota de agua que se les entregó como reforzador.

Con el propósito de replicar el procedimiento más común en los estudios del BIP, en la quinta condición se mantuvieron constantes el programa de 
reforzamiento con agua de IF $2 \mathrm{~s}$, la entrega de comida y la privación de comida, pero se suspendió la privación de agua.

Para determinar la función del agua como controlador del patrón de lengüetazos en presencia de las variables necesarias para establecer el BIP, en la sexta condición experimental se mantuvieron constantes la privación de comida y la entrega periódica de comida, variables necesarias para la ocurrencia del BIP, y se alargó la duración del programa de reforzamiento con agua de un IF $2 \mathrm{~s}$ a un IF $64 \mathrm{~s}$.

En la última condición experimental, se redeterminaron los efectos de entregar agua conforme a un programa de reforzamiento de IF $64 \mathrm{~s}$ con comida añadida en cada intervalo y con privación de comida y de agua. Estas condiciones se probaron por primera vez en la tercera condición experimental del estudio.

En la Figura 1 se presenta un esquema del procedimiento. Los cuadros representan las variables típicas de un programa de reforzamiento operante y los círculos representan las variables necesarias para establecer el BIP. El orden en que se añadieron las variables del BIP al patrón de lengüetazos observado en la línea base se eligió arbitrariamente de entre las posibles permutaciones del orden en que se pudieran añadir las presentes variables.

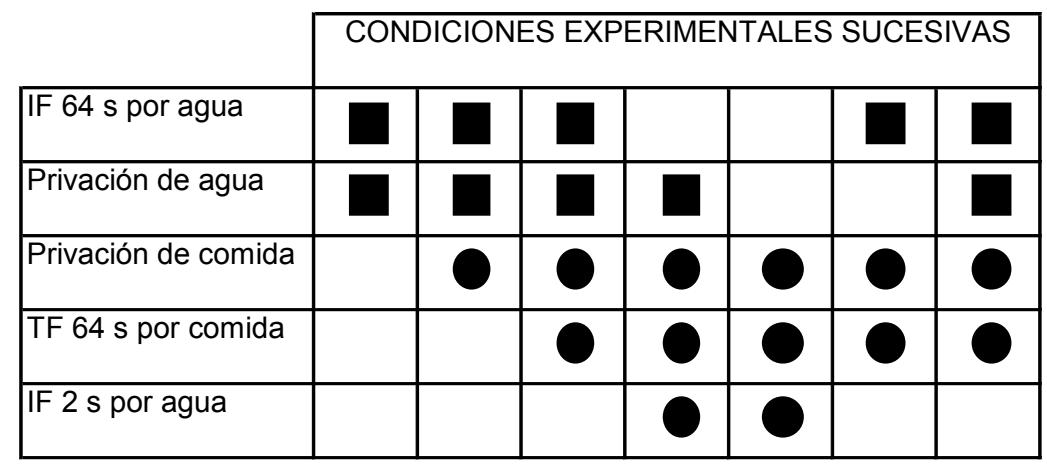

Variables del procedimiento operante de reforzar a intervalos fijos el lengüeteo por agua

Variables de un procedimiento de beber inducido por el programa

Figura 1. Esquema del procedimiento utilizado en el presente estudio. Los cuadros representan las variables típicas de un programa de reforzamiento operante y los círculos representan las variables que controlan el beber inducido por el programa. En cada columna los símbolos señalan la condición en que cada variable estuvo presente. 
Se expuso a los sujetos a la línea base durante 35 sesiones y a las siguientes condiciones durante 20 sesiones cada una. Cada sesión terminó después de una hora o después de 50 entregas de agua o 50 entregas de comida, de acuerdo a las condiciones en las que estuvo vigente el IF $64 \mathrm{~s}$ por agua o el IF $2 \mathrm{~s}$ por agua, respectivamente. Las sesiones se condujeron siete días a la semana y se introdujo a los sujetos a la cámara experimental siempre a la misma hora y en el mismo orden. En las condiciones experimentales en las que se privó de agua a los sujetos se les dio acceso al agua durante $60 \mathrm{mi}-$ nutos, una hora después del final de cada sesión experimental, de tal manera que antes de cada sesión experimental las ratas estuvieron privadas de agua durante 21 horas.

\section{Resultados}

Debido a que el procedimiento del presente estudio consistió en añadir una a una las variables responsables de la ocurrencia del BIP para transformar progresivamente el festón de lengüetazos a un tubo a un patrón en $U$ invertida de la misma variable dependiente durante el intervalo entre reforzadores, fue importante observar la distribución temporal de los lengüetazos durante el intervalo entre reforzadores. En las Figuras 2 y 3 se presenta la distribución temporal de los lengüetazos de cada una de las tres ratas durante dos intervalos contiguos de 64 subintervalos de un segundo cada uno. Se muestran dos intervalos sucesivos para destacar la continuidad en el patrón conductual interrumpido por la intromisión de los eventos experimentales. Para la línea base y para las condiciones experimentales en las que se entregó el agua conforme al programa de reforzamiento IF 64 s, se muestra la distribución temporal de los lengüetazos durante el intervalo fijo por agua. Con el propósito de mantener la comparabilidad entre las condiciones en las que estuvo vigente el programa de reforzamiento con agua de IF $64 \mathrm{~s}$ y de IF $2 \mathrm{~s}$, en el último caso se conceptualizó un ciclo de tiempo repetitivo de $64 \mathrm{~s}$ dentro del cual ocurrió la entrega de comida en el segundo 48. En cada abscisa se señala con una flecha el momento en el que se entregó agua o comida a las ratas. Los datos que se muestran en cada panel de la figura son el promedio de la tasa de lengüeteo de cada rata en las últimas cinco sesiones de exposición a cada condición. Debido a la muerte del sujeto R2 después de completar 11 sesiones de la quinta condición, los paneles correspondientes a este sujeto aparecen en blanco para las últimas dos condiciones.

Contando los paneles de las Figuras 2 y 3 de izquierda a derecha, en el primero se muestra que exponer a los sujetos privados de agua al programa de reforzamiento IF $64 \mathrm{~s}$ por agua resultó en una tasa de lengüeteo relativamente alta al inicio del intervalo, que disminuyó casi a cero aproximadamente 


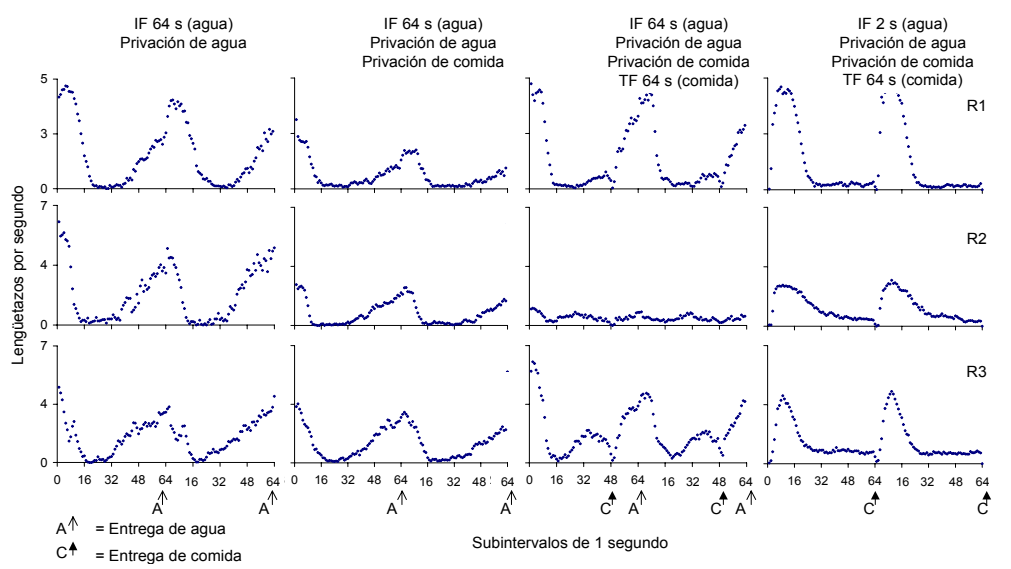

Figura 2. Distribución temporal del lengüeteo para cada una de las tres ratas durante dos intervalos contiguos de 64 subintervalos de un segundo cada uno, para las primeras cuatro condiciones experimentales. En los ejes de las abscisas se señala con flechas el subintervalo en el que se entregó agua o comida a las ratas.

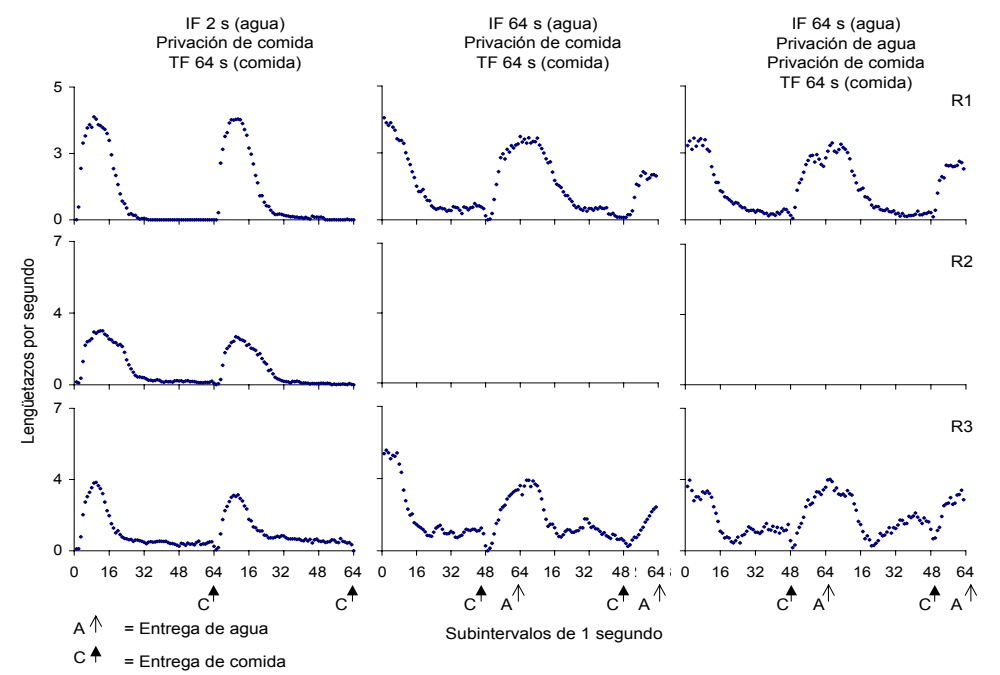

Figura 3. Distribución temporal del lengüeteo para cada una de las tres ratas durante dos intervalos contiguos de 64 subintervalos de un segundo cada uno, para las últimas tres condiciones experimentales. En los ejes de las abscisas se señala con flechas el subintervalo en el que se entregó agua o comida a las ratas. 
en los primeros $16 \mathrm{~s}$ y después aumentó gradualmente hasta la siguiente entrega de agua.

En el segundo panel se muestran los efectos de privar de comida a los sujetos, además de privarlos de agua. Esta operación resultó en una disminución de la tasa de lengüeteo durante todo el intervalo fijo aunque se conservó el aspecto festoneado del lengüeteo hacia la siguiente entrega de agua.

En el tercer panel se muestra el efecto de añadir la entrega de comida, 48 s después de cada entrega agua y manteniendo a los sujetos privados de agua y de comida. Este cambio resultó en un aumento en la tasa de lengüeteo durante el intervalo respecto a la condición previa (en dos de tres sujetos). La tasa de lengüeteo fue alta al inicio del intervalo y disminuyó en los primeros segundos, después aumentó gradualmente hasta la entrega de la comida. Durante la entrega de la comida la tasa de lengüeteo disminuyó a un nivel cercano a cero y después volvió a aumentar hasta la siguiente entrega de agua.

En el cuarto panel se muestra que acortar la duración del programa de entrega de agua de un IF $64 \mathrm{~s}$ a un IF $2 \mathrm{~s}$, con la privación de agua y de comida y el programa de entrega de comida constantes, modificó el patrón de lengüeteo. Mientras que en la condición anterior se observaron festones hacia la comida y hacia el agua, en esta condición la tasa de lengüeteo permaneció constante desde el inicio del ciclo de $64 \mathrm{~s}$ hasta la entrega de la comida. Durante los siguientes $16 \mathrm{~s}$ a la entrega de la comida la tasa de lengüeteo aumentó y después disminuyó hacia la entrega de la siguiente comida, a la manera de una $U$ invertida.

En el quinto panel se muestra que suspender la privación de agua, manteniendo constantes la privación de comida y los programas de entrega de comida y de reforzamiento con agua, controló esencialmente el mismo patrón de $U$ invertida de los lengüetazos al tubo durante el intervalo entre comidas de la condición anterior, aunque la tasa de lengüeteo durante el periodo precedente a la entrega de la comida fue más baja.

En el sexto panel se muestra que el efecto de reinstalar el programa de reforzamiento con agua en un IF $64 \mathrm{~s}$, manteniendo constantes la privación de comida y el programa de entrega de comida, consistió en la reaparición del patrón de festón del lengüeteo por agua observado en la línea base operante. La tasa de lengüeteo observada en esta condición fue más alta que en el procedimiento de BIP implementado en la condición previa.

En el séptimo panel se muestra que cuando se volvió a privar de agua a los sujetos y se mantuvieron constantes la privación de comida y los programas de entrega de comida y de reforzamiento con agua (IF $64 \mathrm{~s}$ ), la tasa de lengüeteo durante el intervalo fue aproximadamente igual que en la condición previa y se mantuvo el festón de lengüeteo hacia la entrega del agua subsiguiente. 


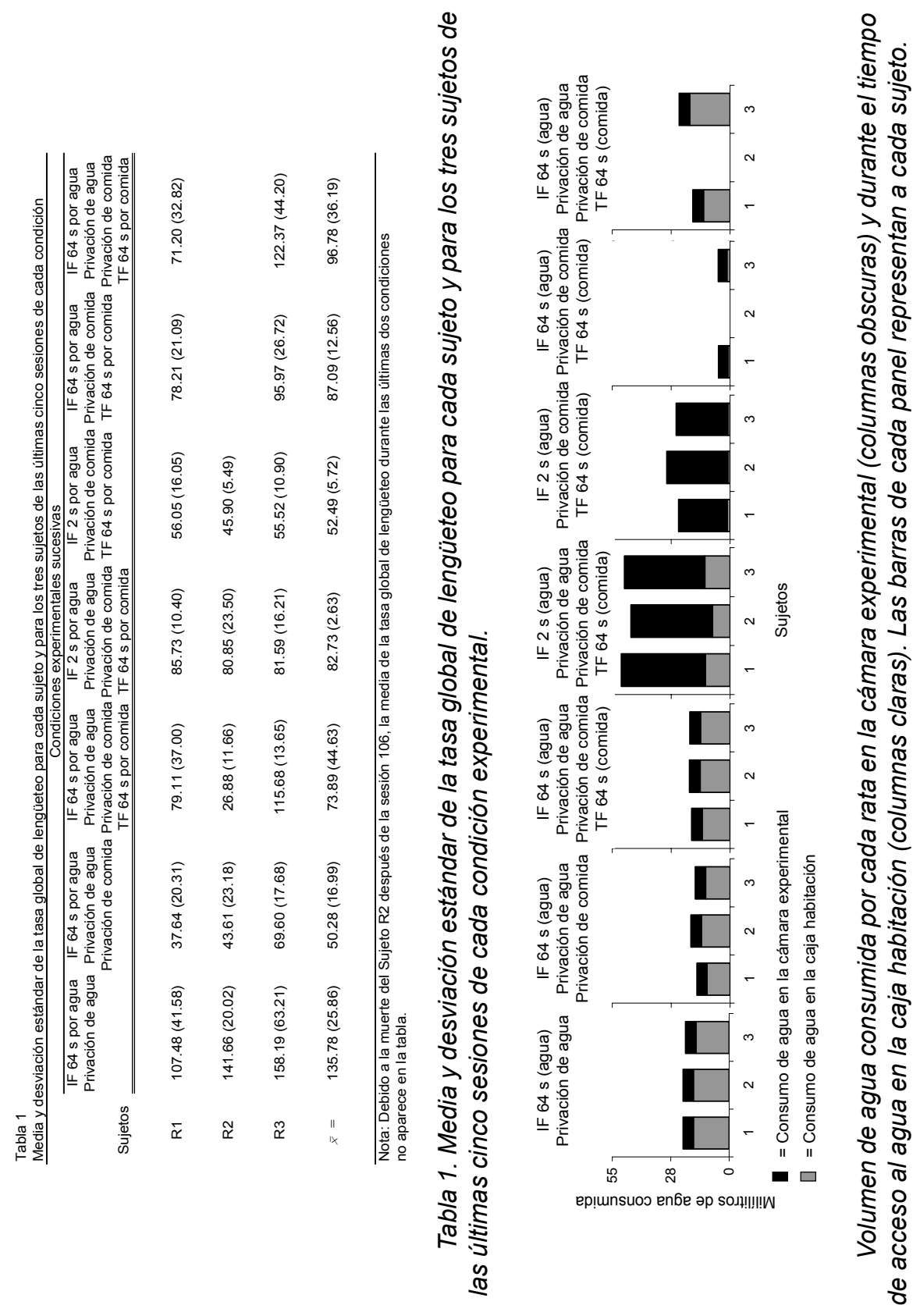


Los datos que se presentan en las Figuras 2 y 3 muestran que la tasa global de lengüetazos varió entre las diferentes condiciones experimentales. En los estudios sobre el BIP la variable dependiente que comúnmente se reporta es la tasa global de lengüeteo. Con fines de comparación de la tasa global de lengüeteo entre las diferentes condiciones del presente experimento en la Tabla 1 se muestran las medias y las desviaciones estándar de la tasa global de lengüeteo por sujeto y de los tres sujetos durante las últimas cinco sesiones de exposición a cada condición del experimento. En comparación con la tasa global de lengüeteo sostenida por el programa de reforzamiento IF $64 \mathrm{~s}$ con privación de agua (primera condición), añadir la privación de comida (segunda condición) resultó en una disminución de la tasa global de lengüeteo. En las condiciones en las que estuvieron presentes el programa de reforzamiento IF $2 \mathrm{~s}$ y la entrega periódica de comida, la tasa global de lengüeteo fue más alta cuando las ratas estaban privadas de comida y de agua (cuarta condición) que cuando sólo estaban privadas de comida (quinta condición). En las condiciones en que se entregó comida periódicamente bajo privación sólo de comida, la tasa global de lengüeteo fue más alta bajo el programa de reforzamiento con agua IF $64 \mathrm{~s}$ (sexta condición) que bajo el programa de reforzamiento con agua IF $2 \mathrm{~s}$ (quinta condición).

En estudios anteriores se ha encontrado que los aumentos o las disminuciones del consumo de agua en la cámara experimental están acompañados por disminuciones o aumentos del consumo de agua en la caja habitación (e.g., Bruner \& Avila, 2002). En la Figura 4 se presenta, para todas las condiciones del experimento, la media del volumen de agua que cada rata consumió en la cámara experimental y durante el tiempo que tuvieron acceso al agua en la caja habitación durante los últimos cinco días de cada condición. Las barras en cada panel representan a cada una de las tres ratas en condiciones sucesivas del experimento. La parte clara de las barras muestra el volumen de agua consumida en la caja habitación y la parte obscura representa el volumen de agua consumida en la cámara experimental. El volumen de agua consumida en la caja habitación estuvo limitado por la duración del periodo de acceso al agua que tuvieron las ratas después de cada sesión experimental, ya sea una hora en las condiciones en las que estuvieron privadas de agua o de 23 horas cuando no estuvieron privadas de agua. El volumen de agua consumida en la cámara experimental dependió de la duración del programa de reforzamiento con agua de IF ya sea 64 ó $2 \mathrm{~s}$. Como se muestra en la Figura 4, el consumo de agua en la caja habitación fue mayor que en la caja experimental en las condiciones en las que se entregó agua conforme a un programa de reforzamiento de IF $64 \mathrm{~s}$, que cuando se entregó agua con un programa de IF $2 \mathrm{~s}$. El único hallazgo intrigante fue la redistribución del volumen de agua consumida entre la caja habitación y la cámara experimental, que se observó en las condiciones en las que se entregó el agua conforme 
al programa de reforzamiento IF $2 \mathrm{~s}$. Cuando los sujetos estuvieron privados de agua y de comida consumieron más agua en la cámara experimental que en la caja habitación. En cambio, cuando los sujetos estuvieron privados sólo de comida consumieron más agua en la caja habitación que en la cámara experimental. No obstante, el volumen total de agua consumida fue casi el mismo en las dos condiciones experimentales.

\section{Discusión}

En el presente estudio se añadieron una a una las variables responsables de la ocurrencia del BIP a una situación típicamente operante. Al transformar el patrón de festón controlado por un programa de reforzamiento de intervalo fijo a un patrón de respuesta en forma de $U$ invertida, típico del BIP, se mostró que las condiciones responsables del BIP producen efectos estudiados con anterioridad en el campo del condicionamiento.

En la primera condición experimental se entregó agua a ratas privadas de agua conforme a un programa de reforzamiento IF $64 \mathrm{~s}$ y se encontró que hubo una tasa alta después de cada entrega de agua. Esta tasa alta se debió a un artefacto del mecanismo empleado para entregar el agua, ya que las ratas debieron lengüetear el tubo varias veces para consumir la gota de agua que se entregó al final de cada intervalo entre reforzadores. No obstante, después de esta tasa alta de lengüeteo al inicio de cada intervalo se encontraron patrones de lengüeteo a la manera de un festón hacia la siguiente entrega de agua, como los que se han reportado en estudios anteriores sobre condicionamiento operante (e.g., Dews, 1970; Ferster \& Skinner, 1957).

Una vez que se contó con un patrón de respuesta reconociblemente operante, se usó como un patrón de referencia sobre el cual se determinaron los efectos de añadir las variables del BIP. Se encontró que privar de comida a los sujetos (segunda condición), además de agua, resultó en que la tasa de lengüeteo al tubo disminuyó globalmente durante el intervalo fijo, relativo a la condición anterior, conservando el aspecto festoneado de la condición previa. Este resultado es consistente con los hallazgos reportados en estudios en los que se reportó que privar de comida a una rata resulta en una disminución del consumo de agua en comparación con su consumo de agua en alimentación libre, esto es, que la privación explícita de comida resulta indirectamente en una privación de agua (e.g., Bolles, 1961). Verplanck y Hayes (1953) demostraron que cuando se da acceso al agua a ratas privadas sólo de agua, éstas consumen más agua que cuando están privadas de agua y de comida. Bolles y Morlock (1960) privaron a ratas de comida y de agua o sólo de agua. Las ratas debían correr en un pasadizo para obtener agua en la caja meta. Encontraron que privar a las ratas de comida y de agua resultó 
en que la velocidad a la que corrían las ratas para llegar a la caja meta fue menor que cuando privaron a las ratas sólo de agua.

En el presente estudio se encontró que mantener a los sujetos privados de agua y de comida, resultó en una disminución de la tasa de respuesta por agua, en comparación con la primera condición en la que sólo estaban privados de agua. Tanto el resultado del presente trabajo como los de los estudios descritos previamente muestran que la privación de comida resulta en la disminución del consumo de agua o de la tasa de emisión de una respuesta procuradora de agua, en lugar de un efecto aditivo de la privación explícita de agua que se suma a la privación indirecta de agua causada por la privación de comida.

En la tercera condición del presente estudio se entregó comida $48 \mathrm{~s}$ después de cada entrega de agua, mientras el agua continuó entregándose conforme a un programa de reforzamiento IF $64 \mathrm{~s}$ y las ratas continuaron privadas de agua y de comida. Se encontró que la tasa de lengüeteo durante el intervalo fijo aumentó respecto a la condición anterior. Este hallazgo sugiere que la entrega de comida concurrentemente a la entrega de agua restableció una tasa alta de lengüeteo por agua. Este resultado es consistente con el hallazgo de que cuando se entrega agua y comida a ratas privadas de agua y de comida, el consumo de agua es mayor que cuando sólo se entrega agua (Verplanck \& Hayes, 1953). En otros estudios también se ha demostrado que dar acceso libre a la comida y al agua a ratas privadas de comida resulta en el restablecimiento del consumo de agua (Ang, McKitrick, Phillips, \& Arnolda, 2001; Morrison, 1968).

Es importante señalar que los hallazgos de que la privación de comida resulta en una disminución en el consumo de agua (e.g., Bolles, 1961; Verplanck \& Hayes, 1953) y de que la entrega de comida a ratas privadas de comida reestablece el consumo de agua (e.g., Ang, et al., 2001) apoyan la propuesta de Bruner y Avila (2002) quienes sugirieron que la privación de comida y la entrega de comida dotan de propiedades reforzantes al agua en los procedimientos de BIP aun cuando una botella con agua se encuentra disponible continuamente en la caja habitación de las ratas.

Introducir la comida en una ubicación constante durante el intervalo fijo de agua resultó en una aceleración de la tasa de lengüeteo hacia la comida y hacia la siguiente entrega de agua. Este resultado es similar al que encontraron Farmer y Schoenfeld (1966) al variar sistemáticamente la ubicación de un estímulo neutral durante un intervalo entre comidas. Los autores concluyeron que el estímulo originalmente neutral funcionó como un reforzador condicionado del patrón de respuestas precedentes y como un estímulo discriminativo del patrón de respuestas subsiguientes. De manera semejante, los resultados del presente estudio parecieron mostrar que la comida funcionó como un reforzador de los lengüetazos previos. Sin embargo, una interpretación 
más parsimoniosa de este efecto es que la entrega de agua al final del intervalo fijo precedente funcionó como un estímulo discriminativo que controló el inicio del festón hacia la siguiente entrega de agua, pero que fue interrumpido por la entrega de comida. Esta última a su vez se convirtió en un segundo estímulo discriminativo de la cercanía temporal del próximo reforzador de agua. El hallazgo de que la comida funcionó como un estímulo discriminativo en el presente estudio es comparable con el de Reid (1968), quien entrenó a ratas a presionar una palanca para obtener comida y posteriormente expuso a los sujetos a extinción. Durante el periodo de extinción entregó comida independientemente de la conducta de las ratas y encontró que, después de cada entrega de comida, las ratas presionaron nuevamente la palanca. Concluyó que la comida podía controlar la ocurrencia de una respuesta a la manera de un estímulo discriminativo.

Cuando se acortó la duración del programa de reforzamiento con agua de un IF $64 \mathrm{~s}$ a un IF $2 \mathrm{~s}$ (cuarta condición), la tasa global de lengüeteo fue similar a la tasa encontrada en el programa de reforzamiento con agua de IF 64 s, vigente en la condición anterior. Sin embargo, el patrón de lengüeteo consistió en que después de la entrega de cada comida, la tasa aumentó y después disminuyó gradualmente hasta la entrega de la siguiente comida, a la manera de una $\mathrm{U}$ invertida. Es importante notar que en el presente trabajo se encontró que los lengüetazos de ratas privadas de agua se distribuyeron en forma de $U$ invertida durante el intervalo entre comidas estuvieran o no privadas de comida. Este hallazgo cuestiona que la distribución de $U$ invertida sea exclusiva del BIP y característica de una tercera clase de conducta (cf., DeCarolis, Myracle, Erbach, Glowa, Flores, \& Riley, 2003; Falk, 1971; Staddon, 1977).

Relativo a resultados de estudios en condicionamiento operante (e.g., Farmer \& Schoenfeld, 1966; Reid, 1968), la distribución temporal del lengüeteo durante la cuarta condición mostró que la comida mantuvo su función de estímulo discriminativo de los lengüetazos que le siguieron pero no fue precedida por el aumento en el lengüeteo observado en la condición previa. Es probable que la disminución en el lengüeteo antes de la comida se deba a que el cambiar el programa de reforzamiento con agua de IF $64 \mathrm{~s}$ a IF $2 \mathrm{~s}$ perturbó la función del periodo siguiente a la última entrega de agua como estímulo discriminativo del inicio del festón hacia la siguiente entrega de agua.

En la literatura sobre el BIP se han considerado diferentes efectos de la entrega de comida de acuerdo a las hipótesis que han apoyado los diferentes autores. Por ejemplo, Clark (1962) consideró que la entrega de la comida reforzaba supersticiosamente los lengüetazos al tubo con agua debido a que éstos eran seguidos cercanamente por la entrega de la comida. Stein (1964) sugirió que la comida era un evocador del beber debido a la sed provocada por la comida seca. Otros investigadores consideraron que la comida se- 
ñalaba un periodo de baja probabilidad de reforzamiento con comida (Falk, 1971) o que era un evento que controlaba la inducción de diferentes conductas entre las entregas sucesivas de comida (Staddon, 1977). Los resultados del presente estudio mostraron que a través de las diferentes condiciones experimentales la entrega de comida funcionó conforme a las predicciones de las diferentes teorías acerca del la naturaleza del BIP e incluso como un estímulo evocador para el beber, dada la cercanía temporal de la entrega de agua. Cabe destacar que los autores de estas distintas hipótesis parecieron enfatizar diferentes relaciones de control entre los eventos experimentales y porciones selectas del intervalo entre comidas que decidieron observar para apoyar a una u otra hipótesis.

En la quinta condición de este estudio, la entrega del agua a las ratas continuó conforme a un programa de reforzamiento de IF $2 \mathrm{~s}$. La entrega de comida a intervalos también estuvo en efecto, así como la privación de comida. A diferencia de la condición anterior, se suspendió la privación de agua. En esta condición experimental estaban presentes todas las variables necesarias para la ocurrencia del BIP, es decir, la privación de comida, la entrega de comida a intervalos de tiempo y la entrega de agua continuamente (cf., Falk, 1961). En esta condición experimental, se encontró esencialmente la misma distribución temporal de lengüetazos al tubo que durante la condición anterior, en la que las ratas todavía estaban privadas de agua. La tasa global de lengüeteo fue menor que en la condición previa. Este resultado sugiere que la privación de comida, aún cuando el agua se encuentra disponible en la jaula habitación de las ratas, produce una privación indirecta de agua que, sin embargo, es menor que la privación producida por 21 horas sin acceso al agua antes de cada sesión experimental.

Después de transformar un procedimiento operante en un procedimiento de BIP, se volvió a alargar el programa de reforzamiento con agua de un IF 2 $\mathrm{s}$ a un IF $64 \mathrm{~s}$ (sexta condición). Las ratas continuaron privadas de comida, pero no de agua y se les entregó comida $48 \mathrm{~s}$ después de cada entrega de agua. La función de $U$ invertida encontrada en la condición anterior, cambió a un festón de lengüeteo controlado por la entrega de agua cada $64 \mathrm{~s}$. Este hallazgo muestra que cuando las variables necesarias para la ocurrencia del BIP estaban presentes, la entrega del agua a intervalos fijos adquirió control temporal sobre el patrón de lengüeteo. El resultado de que el agua controló el patrón de lengüeteo, como en cualquier otro procedimiento operante un reforzador controla el patrón temporal de la emisión de una respuesta, es congruente con el hallazgo reportado por Bruner y Avila (2002) de que el lengüeteo por agua en la situación de BIP se distribuyó a manera de un patrón de festón bajo un procedimiento de reforzamiento con agua IF t $\mathrm{s}$ ( $\mathrm{t}$ $=64,32,16$ y $8 \mathrm{~s}$ ). Tanto los resultados del presente estudio como los de Bruner y Avila (2002) parecen mostrar que, a diferencia de los estudios que 
trataron de condicionar al BIP de manera Pavloviana o como una conducta supersticiosa con procedimientos operantes considerando a la comida como el único reforzador en un procedimiento de BIP (e.g., Wetherington, 1982), caracterizar al agua como el reforzador de la conducta que la procura representa una potencial reducción de la "tercera" clase de conducta a un caso de conducta operante.

Los resultados encontrados en el presente estudio muestran que la distribución de los lengüetazos en una función de $U$ invertida durante el intervalo entre comidas no es distintiva del BIP pues, como se encontró en la cuarta condición del presente estudio, aun cuando los sujetos se encontraban privados de agua se observó dicha distribución. De hecho, el hallazgo de que es posible obtener patrones de festón de la respuesta procuradora de agua bajo las condiciones de BIP en la sexta condición (i.e., privación de comida y entrega periódica de comida) sugiere dos cosas. Primero, que el agua puede ser caracterizada como el reforzador de la conducta que la procura dado que es posible el condicionamiento de un patrón de conducta y; segundo, que el patrón del lengüeteo a la manera de una $U$ invertida reportado en la literatura del BIP (e.g., Killeen, 1975; Staddon, 1977) podría ser un artefacto de la intermitencia con la que se entrega el agua y no un producto del conflicto que se crea en los sujetos al terminar y al anticiparse a cada una de las comidas (Timberlake, 1997). Es decir, puede ser que la distribución de los lengüetazos al tubo con agua a la manera de una $U$ invertida durante el intervalo entre comidas de los procedimientos de BIP sea el producto de que en los estudios previos el agua siempre se entregó conforme a un programa de reforzamiento continuo, sin embargo, al modificar la frecuencia de reforzamiento también se modifica la frecuencia y distribución de la conducta de beber como cualquier otra conducta operante (cf., Ferster \& Skinner, 1957).

En la última condición experimental de este estudio, continuó en efecto el programa de reforzamiento con agua IF $64 \mathrm{~s}$ y el programa de entrega de comida. A diferencia de la condición anterior en la que las ratas sólo estuvieron privadas de comida, en esta condición se les privó también de agua. Esta última condición experimental fue una replicación directa de la tercera condición del estudio. Si bien se mantuvo el patrón de festón por agua en esta condición, la tasa global de lengüeteo fue mayor que en la condición anterior y semejante a la tasa de lengüeteo encontrada cuando las ratas fueron expuestas a estas mismas manipulaciones experimentales. Estos datos confirman que bajo una privación simultánea de agua y de comida disminuye el consumo de agua y que la entrega de comida restablece dicho consumo (e.g., Verplanck \& Hayes, 1953).

Un resultado intrigante del presente estudio es que en la condición en la que se reprodujo el procedimiento más común de BIP (IF $2 \mathrm{~s}$ por agua, privación de comida y TF $64 \mathrm{~s}$ de comida) se encontró que las ratas consu- 
mieron casi la mitad de su ración diaria de agua en la cámara experimental y la otra mitad en la caja habitación. En la condición inmediata anterior en la que estaban presentes las variables del BIP pero también había privación de agua, la mayor parte del consumo diario de agua ocurrió dentro de la cámara experimental. Aunque el periodo de acceso al agua en la caja habitación fue diferente entre las dos condiciones, las ratas consumieron aproximadamente la misma cantidad de agua por día. Este resultado sugiere que un procedimiento de BIP lejos de generar consumo excesivo en la cámara experimental (cf., Falk, 1998), resulta en que las ratas distribuyen el volumen de agua que consumen por día entre la cámara experimental y la caja habitación (cf., Bruner \& Avila, 2002).

El procedimiento utilizado en el presente estudio permitió transformar progresivamente un patrón operante reforzado por agua en un patrón característico del BIP y mostró el efecto de añadir una a una las variables necesarias para la ocurrencia del BIP. Los resultados del estudio mostraron que hubo una transición progresiva entre ambos patrones de respuesta y que en ningún momento las variables independientes dejaron de reflejar efectos que han sido ampliamente estudiados en experimentos anteriores en el área del condicionamiento operante. Por esta razón, parece injustificado el categorizar al BIP como un fenómeno extraño en el área del condicionamiento operante. Más bien parece que la combinación simultánea de la privación de comida, la entrega intermitente de comida y la disponibilidad del agua conforme a un programa de reforzamiento continuo generan que las ratas sin privación explícita de agua "beban" agua en cantidades substanciales. El fenómeno del BIP es contraintuitivo porque las ratas beben a pesar de tener acceso libre al agua en sus cajas habitación. Este carácter anómalo ha sido la variable que ha controlado que diferentes teóricos lo clasifiquen como un fenómeno inexplicable por los principios establecidos en el análisis experimental de la conducta. En este sentido es interesante notar que la aparición de fenómenos anómalos en Teoría de la Conducta da lugar al dilema reconocido por Cabrer, Daza, y Ribes (1975) relativo a que los teóricos tengan que elegir entre categorizar estos fenómenos como nuevos para el análisis experimental de la conducta o reconocer que simplemente se tratan de una nueva combinación de antiguos parámetros.

\section{REFERENCIAS}

Allen, J. D. \& Porter, J. H. (1977). Sources of control over schedule-induced drinking produced by second-order schedules of reinforcement. Physiology and Behavior, $18,853-863$. 
., \& Araize, R. (1975). Schedule-induced drinking as a function of percentage reinforcement. Journal of the Experimental Analysis of Behavior, 23, 223-232.

Ang, K. K., McKitrick, D. J., Phillips, P. A., \& Arnolda, L. F. (2001) Time of day and acces to food alter water intake in rats after water deprivation. Clinical \& Experimental Pharmacology \& Physiology, 28, 764-767.

Bolles, R. C. (1961) The interaction of hunger and thirst in the rat. Journal of Comparative and Physiological Psychology, 54, 580-584.

—, \& Morlock, H. (1960). Some asymmetrical drive summation phenomena. Psychological Reports, 7, 373-378.

Breland, K., \& Breland, M. (1961). The misbehavior of organisms. American Psychologist, 16, 681-684.

Bruner, C. A., \& Avila, R. (2002). Adquisición y mantenimiento del palanqueo en ratas sin privación explícita del reforzador. Revista Mexicana de Análisis de la Conducta, 28, 107-130.

Cabrer, F., Daza, B. C., \& Ribes, E. (1975) Teoría de la Conducta: ¿Nuevos conceptos o nuevos parámetros?, Revista Mexicana de Análisis de la Conducta, 1, 191212.

Clark, F. C. (1962). Some observations of the adventitious reinforcement of drinking under food reinforcement. Journal of the Experimental Analysis of Behavior, 5, 61-63.

Corfield-Sumner, P. K., Blackman, D. E., \& Stainer, G. (1977). Polydipsia induced in rats by second-order schedule of reinforcement. Journal of the Experimental Analysis of Behavior, 27, 265-273.

DeCarolis, N. A., Myracle, A., Erbach, J., Glowa, J., Flores, P., \& Riley, A. L. (2003) Strain-dependent differences in schedule-induced polydipsia: an assessment in Lewis and Fischer rats. Pharmacology, Biochemistry and Behavior, 74, 755-763.

Dews, P. B. (1970). The theory of fixed-interval responding. En: W. N. Schoenfeld (Ed.), The Theory of Reinforcement Schedules. (pp. 43-61). New York: AppletonCentury-Crofts.

Falk, J. L. (1961). Production of polydipsia in normal rats by an intermittent food schedule. Science, 133, 195-196.

—_. (1964). Studies on schedule-induced polydipsia. En: M. J. Wayner (Ed.), Thirst: First International Symposium on thirst in the regulation of body water. (pp. 95116). New York: Pergamon Press.

— . (1966). Schedule-induced polydipsia as a function of fixed interval length. Journal of the Experimental Analysis of Behavior, 9, 37-39.

- (1969). Conditions producing psychogenic polydipsia in animals. Annals of the New York Academy of Sciences, 157, 569-593.

- (1971). The nature and determinants of adjunctive behavior. Physiology and Behavior, 6, 577-588.

- (1998). Addictive behavior with and without pharmacologic action: critical role of stimulus control. En: C. L. Wetherington \& J. L. Falk (Eds.), Laboratory Behavioral Studies of Vulnerability to Drug Abuse (pp. 134-153). NIDA Research Monograph 169. 
Farmer, J., \& Schoenfeld, W. N. (1966). Varying temporal placement of an added stimulus in a fixed-interval schedule. Journal of the Experimental Analysis of Behavior, 9, 369-375.

Ferster, C. B., \& Skinner, B. F. (1957). Schedules of Reinforcement. Englewood Cliffs, $\mathrm{NJ}$ : Prentice-Hall.

Flory, R. K. (1971). The control of schedule-induced polydipsia: Frequency and magnitude of reinforcement. Learning and Motivation, 2, 215-227.

Hawkins, T. D., Schrot, J. F., Githens, S. H., \& Everett, P. B. (1972). Schedule-induced polydipsia: An analysis of water and alcohol ingestion. En R. M. Gilbert \& J. D. Keehn (Eds.), Schedule effects: Drugs, drinking and aggression. (pp. 95-128) Toronto: University of Toronto Press.

Keehn, J. D. (1979). Schedule-induced polydipsia, schedule-induced drinking, and body weight. Bulletin of the Psychonomic Society, 13, 78-80.

Killeen, P. (1975). On the temporal control of behavior. Psychological Review, 82 , 89-115.

Morrison, S. D. (1968). Regulation of water intake by rats deprived of food. Physiology and Behavior, 3, 75-81.

Mowrer, R. R., \& Klein, S. B. (1989). Traditional learning theory and the transition to contemporary learning theory. En: S. B. Klein, \& R. R. Mowrer (Eds.), Contemporary Learning Theories: Pavlovian Conditioning and the Status of Traditional Learning Theory (pp. 3-17). Hillsdale, NJ: Lawrence Erlbaum Associates.

Reid, R. L. (1968). The role of the reinforcer as a stimulus. En: A. C. Catania (Ed.), Contemporary Research in Operant Behavior (pp 67-70). Illinois: Scott, Foresman and Company.

Roper, T., \& Nieto, J. (1979). Schedule-induced drinking and other behavior in the rat, as a function of body weight deficit. Physiology \& Behavior, 23, 673-678.

Segal, E. F., \& Oden, D. L. (1969). Schedule-induced polydipsia: effects of providing an alternate reinforced response and of introducing a lick-contingent delay in food delivery. Psychonomic Science, 15, 153-154.

Staddon, J. E. R. (1977). Schedule-induced behavior. En W. K. Honig, \& J. E. R. Staddon (Eds.), Handbook of Operant Behavior (pp. 125-152). Englewood Cliffs, $\mathrm{NJ}$ : Prentice-Hall.

Stein, L. (1964). Excessive drinking in the rat: Superstition or thirst?. Journal of Comparative and Physiological Psychology, 58, 237-242.

Timberlake, W. (1993). Behavior systems and reinforcement: an integrative approach. Journal of the Experimental Analysis of Behavior, 60, 105-128.

_ (1997). An animal-centered, causal-system approach to the understanding and control of behavior. Applied Animal Behaviour Science, 53, 107-129.

- (2001). Motivational modes in behavior systems. En: R. R. Mowrer \& S. B. Klein (Eds.), Handbook of Contemporary Learning Theories (pp. 155-209). Mahwah, $\mathrm{NJ}$ : Lawrence Erlbaum Associates.

Verplanck, W., \& Hayes, J. (1953). Eating and drinking as a function of maintenance schedule. Journal of Comparative and Physiological Psychology, 46, 327-333.

Wetherington, C. L. (1982). Is adjunctive behavior a third class of behavior?. Neuroscience and Biobehavioral Reviews, 6, 329-350. 